\title{
Quantitative Analysis of the Banned Food Dye Sudan-1 Using Surface Enhanced Raman Scattering with Multivariate Chemometrics ${ }^{\dagger}$
}

\author{
William Cheung, Iqbal T. Shadi, Yun Xu, and Royston Goodacre* \\ School of Chemistry, Manchester Interdisciplinary Biocentre, University of Manchester, 131 Princess Street, \\ Manchester, M1 7DN United Kingdom
}

Received: September 14, 2009; Revised Manuscript Received: January 8, 2010

\begin{abstract}
Sudan-1 has been used for coloring food. However, recent alarms worldwide about the carcinogenic and mutagenic properties of azo-compounds have led to concerns over their human consumption. In the U.K. in 2005 , over 570 products were found to be contaminated with the azo dye Sudan-1 and this and the health risks associated with this dye resulted in the subsequent international ban of this additive in all foodstuff, at all levels, relating to human consumption. These incidents have also necessitated the need for high throughput low cost reliable approaches for the detection and quantification of food contaminated by such azo compounds. While there are a small number of analytical techniques that can be considered portable, many lack sensitivity. By contrast, we show that employing a portable Raman spectrometer, using surface enhanced Raman scattering (SERS), can provide good sensitivity, such that Sudan-1 can be quantified in a complex food matrix reliably over the range of $10^{-3}$ to $10^{-4} \mathrm{~mol} \mathrm{~L}^{-1}$. We also demonstrate that a variety of multivariate approaches including principal components analysis (PCA), partial least-squares (PLS) regression, artificial neural networks (ANNs), and support vector regression (SVR) can be employed for the chemical analysis of this dye in a quantitative manner. Compared to the commonly used univariate approaches, where the area under a single band in assessed, the advantage of using multivariate approaches is that these algorithms can analyze the full spectra directly and the laborious task of selecting and integrating marker appropriate quantitative spectral bands can be avoided thus greatly simplifying and speeding up data analysis.
\end{abstract}

\section{Introduction}

Sudan-1 (1-phenylazo-2-naphthol; Figure 1) is a synthetic azo compound with orange red appearance, widely used as a coloring agent with many commercial applications. It is commonly found in printing ink, color waxes, oils, petrol, solvents, plastics and polishes, as well as foodstuffs. ${ }^{1}$ However, azo dyes have demonstrated significant carcinogenic and mutagenic properties, inducing tumors in the liver and bladder of mice, rats, and rabbits. ${ }^{1-7}$ It has therefore been classified as a third category carcinogen by the international agency for research on cancer (IARC).

However despite this, several high profile international incidents involving the use of Sudan-1 were documented in the past decade. In India in 2003 Sudan-1 was detected in hot chilli products resulting in the U.K.'s food standard agency (FSA) to implement measures for the detection of Sudan-1 for all chilli products originating from India. In China in 2005, Sudan-1 was found in numerous foodstuffs from chilli oil and powder, chicken fast food shops, and turnip pickles causing widespread panic. ${ }^{8}$ In the same year in the U.K., a Worcestershire sauce product was found to be contaminated by Sudan-1, which was latter traced back to adulterated chilli powder used in its production. This product was widely used in many supermarket products such as pizzas and in numerous ready meal products, leading to over 570 products having to be taken off the shelves. More recently Sudan-1 has also been detected in several dried spices products in South Africa forcing them to implement measures similar to India, China, and U.K. for the detection of

\footnotetext{
†Part of the "Martin Moskovits Festschrift".

* To whom correspondence should be addressed. E-mail: roy.goodacre@ manchester.ac.uk. Tel: +44 (0) 1613064480.
}

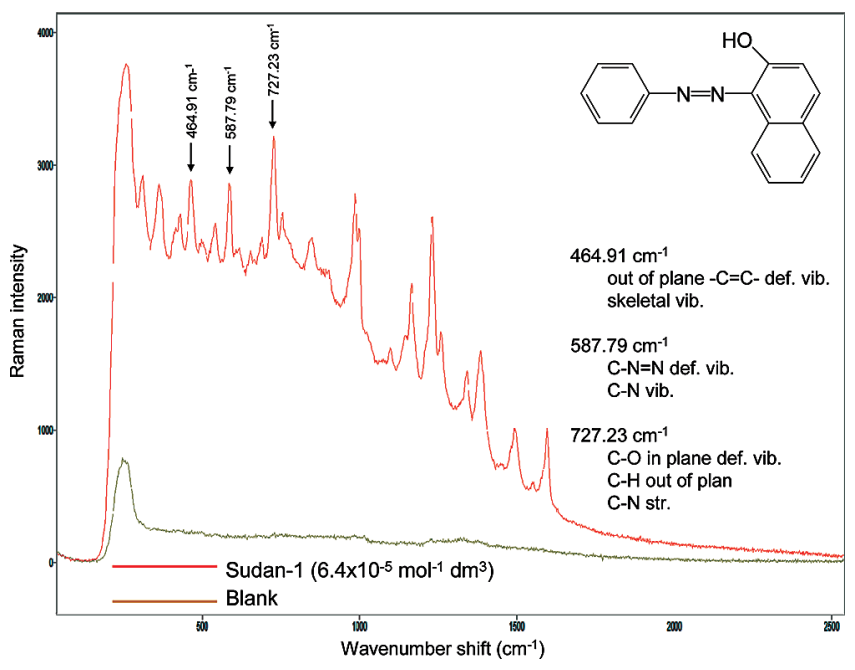

Figure 1. Raw SERS spectrum of Sudan-1 $\left(6.4 \times 10^{-5} \mathrm{~mol}^{-1} \mathrm{dm}^{3}\right)$ with a blank of colloid. Inset is the chemical structure of this azo dye, with tentative assignments for the three highlighted bands, where vib. $=$ vibration, def. $=$ deformation, and str. = stretch.

Sudan-1 within their foodstuffs. Despite the international ban, these incidents have highlighted the need for a rapid, low cost, reliable method for the detection of Sudan-1 in contaminated foods.

Extensive literature is available for the detection of Sudan-1 in contaminated foodstuffs, most of which involve the use of liquid chromatography-based instrumentation for chromatographic separation of Sudan-1 from its food matrix and subsequent detection by various detectors. These include HPLC- 
UV/vis, ${ }^{9,10}$ HPLC fluorimetry, ${ }^{11}$ flow injection chemiluminescence, ${ }^{12}$ HPLC/APCI-MS/MS, ${ }^{13,14}$ and HPLC/ESI-MS/MS..${ }^{15,16}$ In addition, a novel method for sample extraction has also been reported using molecularly imprinted solid phase extraction, ${ }^{17}$ and the use of a glass carbon electrode has also been applied for the detection of Sudan-1 in foodstuffs. ${ }^{8}$

These LC-based methods mentioned for the detection of Sudan-1 are highly robust, reproducible, have relatively high sample throughput through automation, and have very good detection limits. However, they also have some serious draw backs, including sample pretreatment and extractions which can be labor intensive; they are also expensive and not currently portable. The direct analysis of Sudan-1 within the food matrix without sample preparation would hence be very desirable.

Raman spectroscopy is a physicochemical technique, which like the other vibrational spectroscopic method infrared absorption spectroscopy gives chemical information on the molecular make up of a molecule in a nondestructive manner. ${ }^{18}$ Raman spectroscopy offers several advantages over mid-IR and nearIR spectroscopy; little or no sample preparation is required, as water is a weaker Raman scatterer no special accessories are needed for measuring aqueous solutions, and recently there have been many manufacturers producing small portable instrumentation. In combination, this makes this technique ideal for both qualitative and quantitative applications that involve the analysis of organic and inorganic chemicals. A main drawback of Raman spectroscopy is its low sensitivity. Raman scattering only comprises a very small fraction of those photons that are scattered, about 1 in $10^{7}$, of the incident photons; the rest are Rayleigh scattered. ${ }^{19}$ However, such weak Raman effects can be significantly amplified by coupling the analyte molecules to the surfaces of nanometer-sized nanometal structures, a technique termed surface enhanced Raman scattering (SERS). ${ }^{20}$ Under normal conditions enhancements are on the order of $10^{4}-10^{6},{ }^{21}$ as demonstrated for bacterial identification ${ }^{22}$ and for DNA analysis, ${ }^{23}$ but for certain molecules factors up to $10^{14}$ or even greater have been observed leading to the idea of single molecule detection. ${ }^{24,25}$

The objectives of this study were to combine SERS with chemometric analysis ${ }^{26,27}$ for the detection and quantification of Sudan-1; the advantages of multivariate analysis over peak peaking (univariate) shall also be explored. In addition, we shall demonstrate the use of portable Raman spectroscopy for the quantification of Sudan-1 combined with SERS and chemometrics and further show that good signals from Sudan-1 can be generated when this azo dye is spiked into the complex food matrix of chilli powder.

\section{Experimental Section}

Reagents. The following reagents were provided as indicated and used without further purification: Sudan-1, poly (L)-lysine hydrobromide, $\left(\mathrm{M}_{\mathrm{r}}: 4000-15,000\right)$, gold tetrachloride, trisodium citrate were all of analytical grade, purchased from Sigma Aldrich Ltd. (Gillingham, U.K.). Hydrochloric acid was purchased from Fisher Scientific (Loughborough, U.K.). Chilli powder was bought from a local supermarket.

Raman Instrumentation. Spectra were collected using an Ahura defender system (Ahura scientifics, Wilmington, MA) which is a hand-held, portable Raman spectrometer (using back scattered geometry). This instrument has a built in $4 \mathrm{~mL}$ sample chamber which provided up to $300 \mathrm{~mW}$ laser power at $785 \mathrm{~nm} \pm 0.5 \mathrm{~nm}$ laser excitation and $2 \mathrm{~cm}^{-1}$ line width. All spectra were collected with an integration time of $5 \mathrm{~s}$ with the power setting set to low $(30 \mathrm{~mW})$. The spectrometer has a monochromator spectral range of 781-1014 nm, a Raman spectrum range of $250-2875 \mathrm{~cm}^{-1}$, and a spectral resolution of $7-10.5 \mathrm{~cm}^{-1}$ (full width at half-maximum (fwhm)) across the range. Built into the Ahura spectrometer are Rayleigh rejection filters of OD 7, a silicon CCD 2048 pixels detector in direct dispersive detection mode, and a dispersion mode which is a single pass spectrometer (1200 grooves/mm blazed at 900 $\mathrm{nm})$.

Surface Enhanced Raman Scattering. A gold colloid solution was prepared according to ref 18. All glassware was soaked in aqua regia $\left(\mathrm{HNO}_{3}: \mathrm{HCl} 1: 3 v / v\right)$ overnight. The colloid was prepared by reduction of $\mathrm{HAuCl}_{4}$ by sodium citrate: 250 $\mathrm{mL}$ of $1 \mathrm{mM} \mathrm{HAuCl} \mathrm{H}_{4}$ was first brought to the boil, after the addition of $25 \mathrm{~mL}$ of $38.8 \mathrm{mM}$ sodium citrate, the mixture was allowed to boil for a further $30 \mathrm{~min}$. The solution was then left to cool before use.

Aggregation of the citrate-reduced gold colloid particles was induced by poly(L)-lysine. The overall composition of the SERS solution was as follows: $1 \mathrm{~mL}$ of the gold colloid was diluted with $1 \mathrm{~mL}$ of ultra pure water, followed by the addition of 150 $\mu \mathrm{L}(0.01 \%)$ of poly(L)-lysine solution, $150 \mu \mathrm{L}$ of sample solution, and finally $35 \mu \mathrm{L}$ of the $\mathrm{pH}$ modifier (vide infra). The solution was vortex for $3 \mathrm{~s}$ between each successive addition. To allow for aggregation to occur, the solution was allowed to rest within the sample chamber for 1 min before spectral acquisition.

pH Profiling of SERS of Sudan-1. In order to determine the optimal $\mathrm{pH}$ which gave the largest SERS response, a stock solution of Sudan-1 $\left(1 \times 10^{-3} \mathrm{~mol} \mathrm{~L}^{-1}\right)$ was used for $\mathrm{pH}$ profiling. In $\mathrm{pH}$ profiling the $\mathrm{pH}$ of the SERS sample was adjusted between $\mathrm{pH} 1.4$ to $\mathrm{pH} 5.3$ by adding $35 \mu \mathrm{L}$ of a $\mathrm{pH}$ acid modifier with the appropriate $\mathrm{pH}$ into the solution. Three replicate SERS spectra were taken at each $\mathrm{pH}$. Following this the peak areas of the vibrational band at $725 \mathrm{~cm}^{-1}$ were plotted as a function of $\mathrm{pH}$ in order to find the maximum SERS response. This was found to be 2.0 (vide infra) and was used for all future SERS analyses.

Extraction of Sudan-1 from Spiked Chilli Powder. To evaluate the viability for the detection of Sudan-1 in a common complex foodstuff, we used chilli powder as the test matrix; this was chosen as chilli powder has been found in the past to be contaminated with Sudan- $1 .{ }^{8}$ In order to have accurate levels of Sudan-1 in chilli, methanol extractions from unadulterated chilli were prepared. This involved measuring $0.1 \mathrm{~g}$ of chilli powder and transferring this to a Falcon test tube to which 10 $\mathrm{mL}$ of analytical grade methanol was added and vortexed for $10 \mathrm{~min}$. After, this the solution was then allowed to stand for another $5 \mathrm{~min}$. Following this the brownish red supernatant was carefully removed using a pipet and used as the matrix into which Sudan-1 was to be spiked.

Known solutions of the dye dissolved in water were used to spike chilli powder to give a series of Sudan-1 standards with the following concentration range: $2 \times 10^{-3}, 1 \times 10^{-3}$, $8 \times 10^{-4}, 4 \times 10^{-4}, 2 \times 10^{-4}$, and $1 \times 10^{-4} \mathrm{~mol} \mathrm{~L}^{-1}$. Six samples were prepared for each concentration, and each sample was measured three times by SERS. Note that for actual concentrations of the dye being analyzed by SERS one should be aware that these are 15.56 times more dilute than the stock. This is because, for example, in our sample preparation $1 \mathrm{~mL}$ of sol is diluted with $1 \mathrm{~mL}$ of $\mathrm{H}_{2} \mathrm{O}$ followed by aggregation by $150 \mu \mathrm{L}$ of poly(L)-lysine and $150 \mu \mathrm{L}$ of the analyte and $35 \mu \mathrm{L}$ of acid/base. 


\section{Data Analysis}

For univariate analysis we performed a classical linear regression analysis between the areas under the curve (AUC) of the three selected vibrational bands within the SERS spectrum at 464.91, 587.79 , and $727.23 \mathrm{~cm}^{-1}$ (peak apexes). These three bands were chosen as they appeared to have relatively high intensity with little or no background interference. Prior to regression, for each sample the AUCs from the three replicate SERS spectra from the six identical solutions were averaged prior to the analysis. Three regression models were built, one for each vibration band.

For multivariate analysis, the data set were baseline corrected by using asymmetric least-squares $\left(\mathrm{ALS}^{28}\right)$, an adaptive baseline estimation algorithm. The unsupervised learning algorithm principal components analysis (PCA) ${ }^{26,29,30}$ was used to visualized any natural variations within the data set.

Prior to multivariate regression the three replicated spectra SERS spectra from the six identical solutions were averaged prior to the analysis to reduce unavoidable variation caused during collection. For multivariate regression we employed partial least-squares (PLS) regression ${ }^{26,29,31,32}$ to establish a multivariate linear regression model between the whole baseline corrected Raman spectra and the concentrations of the Sudan- 1 in the solution. Considering the fact that the response of SERS intensity with the concentration of the analyte may not necessarily be linear, we also employed two nonlinear methods: $\varepsilon$-support vector regression (SVR) programmed in Matlab ${ }^{33}$ and artificial neural networks (ANNs) using an in house program. ${ }^{34}$ For ANN analysis a multilayer perceptron with a topology of 650-8-1 (650 input Raman scatters, eight nodes in the hidden layer and a single output node for the concentration of Sudan-1) was used employing a learning rate of 0.2 and a momentum of $0.8 .^{35}$

For all three multivariate methods the performances of these three models on our data set was compared. The generalization performance of the multivariate regression models were assessed by using a double cross-validation scheme as detailed in refs 36-38. Each time we left out all of the six samples of one concentration which was used as the blind test set and the remaining samples were used as the training set. The model parameters, e.g., the number of PLS components, the kernel parameters of $\varepsilon$-SVR, etc., were tuned by using another inner $k$-fold cross-validation procedure on the training set only, where $k$ is the number of concentration levels in the training set. Following this the model was built on the full training set using the optimal model parameter found by the inner cross-validation and used to predict the concentration of the samples in the test sets.

This was repeated until all the samples of each concentration level have been left out and predicted once and the root-meansquare error of cross-validation (RMSECV) as well as the predictive squared correlation coefficient $\left(Q^{2}\right)^{26}$ was calculated using the samples in the test sets according to the following two equations:

$$
\begin{gathered}
\text { RMSECV }=\sqrt{\frac{\sum_{i}\left(y_{i}-\hat{y}_{i}\right)^{2}}{n}} \\
Q^{2}=1-\frac{\sum_{i}\left(y_{i}-\hat{y}_{i}\right)^{2}}{\sum_{i}\left(y_{i}-\bar{y}\right)^{2}}
\end{gathered}
$$

where $n$ is total number of samples used for testing; $y_{i}$ is the concentration of sample $i(i=1,2,3, \ldots, n) ; \hat{y}_{i}$ is the predicted

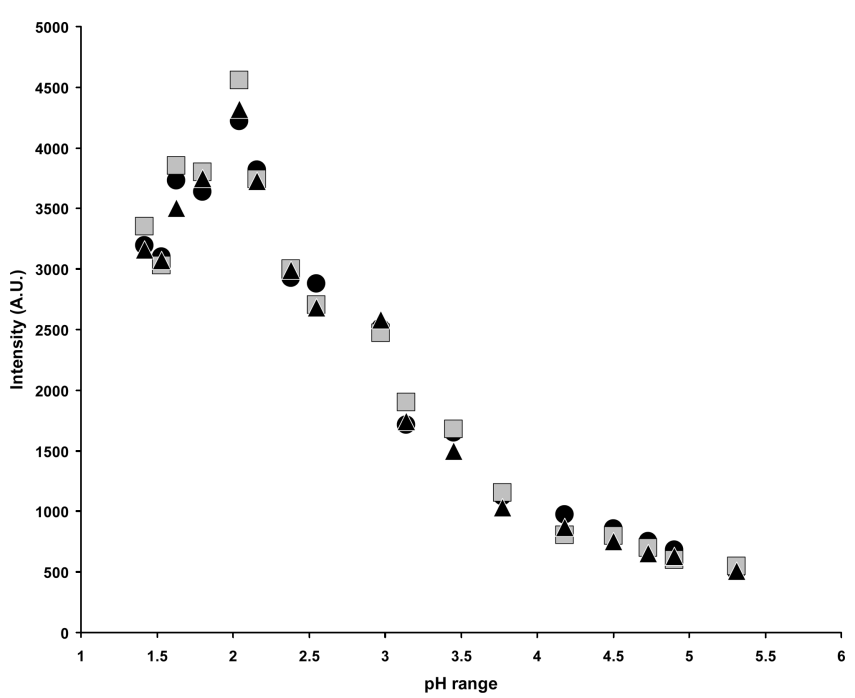

Figure 2. Peak area of the vibrational band at $725 \mathrm{~cm}^{-1}$ plotted against the $\mathrm{pH}$ profile from $\mathrm{pH} 1.4$ to 5.3. The concentration of Sudan-1 in this preliminary experiment was $6.4 \times 10^{-5} \mathrm{~mol}^{-1} \mathrm{dm}^{3}$, and the data were collected in triplicate.

concentration of sample $i$, and $\bar{y}$ is the mean of the concentrations of all the samples used for testing.

RMSECV and $Q^{2}$ are the 2 most used and unbiased metrics to assess the predictive ability of a predictive model in regression analysis. Generally speaking, RMSECV gives an unbiased estimation of the error of the prediction when the model is used to predict the concentration of an unknown sample. Smaller the RMSECV is, the better the model is. Also, $Q^{2}$ is a scale independent, and also unbiased, metric to quantify the predictive ability of the model with values similar to the commonly used squared correlation coefficient $R^{2}$ in univariate regression analysis. The closer the value of $Q^{2}$ is to 1 , the better the model is.

\section{Results and Discussion}

SERS and pH Profiling. A typical SERS spectrum of the pure Sudan-1 dye is shown in Figure 1 along with this azo dye's chemical structure. As can be seen the SERS spectrum is information-rich and the main bands that were used for quantification are 464.91, 587.79, and $727.23 \mathrm{~cm}^{-1}$ and these bands have been tentatively assigned in this figure. ${ }^{39,40}$

It has been shown that the SERS response of many analytes is dependent on the $\mathrm{pH}$ of the solution in which the analyte resides, ${ }^{41,42}$ therefore we conducted a " $\mathrm{pH}$ profile" of the Sudan-1 in order to optimize the signal enhancement. The $\mathrm{pH}$ was therefore adjusted from $\mathrm{pH} 1.4$ to $\mathrm{pH} 5.3$ and SERS measurements taken. Using the strong band at $727.23 \mathrm{~cm}^{-1}$, we constructed a pH profile (Figure 2), and it can be clearly seen that there is a general trend of decreasing intensity toward neutral $\mathrm{pH}$ and that it appears that at a $\mathrm{pH}$ of 2.0 the highest SERS response was seen. Therefore these conditions were used for the quantitative analysis of Sudan-1 in chilli powder.

Assessment of Spiked Chilli Solutions Using Univariate Analysis. As detailed above $2 \times 10^{-3}, 1 \times 10^{-3}, 8 \times 10^{-4}$, $4 \times 10^{-4}, 2 \times 10^{-4}$, and $1 \times 10^{-4} \mathrm{~mol} \mathrm{~L}^{-1}$ Sudan- 1 was spiked into a methanol extract from chilli powder. Chilli powder was chosen as it has been found to be contaminated by Sudan-1; note we took preliminary spectra of the chilli powder and extract and no bands belong to Sudan-1 were seen (data not shown).

In order to see if the SERS signal from Sudan-1 was reproducible enough to allow its quantification the averaged peak areas of the three vibrational bands at 464.91, 587.79, and 727.23 


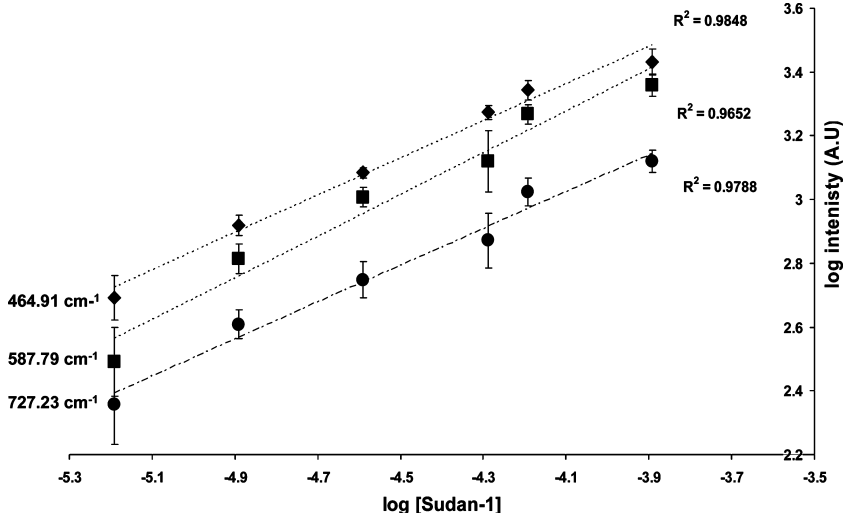

Figure 3. Peak area of three different vibrational bands plotted against the $\log _{10}$ of concentration of Sudan-1; this is equivalent to a concentration range of $6.4 \times 10^{-6}$ to $1.3 \times 10^{-4} \mathrm{~mol}^{-1} \mathrm{dm}^{3}$. The $R^{2}$ values are also shown for the three different bands. The average peak areas for three measurements are shown and the error bars show standard deviations.

TABLE 1: Comparison between Univariate Calibration and Multivariate Chemometric Methods for the Quantification of Sudan-1 from SERS

\begin{tabular}{lccccccc}
\hline & \multicolumn{3}{c}{$\begin{array}{c}\text { univariate analysis from } \\
\text { peak areas }\end{array}$} & & \multicolumn{3}{c}{$\begin{array}{c}\text { multivariate chemometric } \\
\text { methods }\end{array}$} \\
\cline { 2 - 4 } & $\begin{array}{c}464.91 \\
\mathrm{~cm}^{-1}\end{array}$ & $\begin{array}{c}587.79 \\
\mathrm{~cm}^{-1}\end{array}$ & $\begin{array}{c}727.23 \\
\mathrm{~cm}^{-1}\end{array}$ & & PLSR & SVR & $\mathrm{ANN}^{a}$ \\
\hline RMSECV & 0.127 & 0.134 & 0.137 & & 0.123 & 0.095 & 0.084 \\
$Q^{2}$ & 0.911 & 0.906 & 0.903 & & 0.916 & 0.961 & 0.977
\end{tabular}

${ }^{a}$ The values are the averages of 10 independent neural networks.

$\mathrm{cm}^{-1}$ were plotted against the $\log$ of the concentrations of Sudan-1 spiked into the chilli powder (Figure 3). Linear regression analysis was carried out on the areas of each of the selected vibration band in these $\log -\log$ plots and showed very good correlation with the concentration and resulted in the $R^{2}$ values of $0.985,0.965$, and 0.979 respectively for 464.91, 587.79 , and $727.23 \mathrm{~cm}^{-1}$ (Figure 3). As detailed in the data analysis section, these AUC for the bands were also assessed by using a double cross-validation scheme and the RMSECV and $Q^{2}$ are detailed in Table 1.

Principal Components Analysis (PCA). While the RMSECV and $Q^{2}$ values for the univaraite analysis are encouraging this is some room for improvement. We therefore decided to assess the spectra employing multivariate analyses. Initially PCA was used on the SERS spectra from all six Sudan-1 concentrations along with a blank (methanol extract from the chilli powder). The first two principal components (PCs) captured over $88 \%$ of total variance of the data, and the scores plot of PC1 and PC2 are shown in Figure 4A. It can be seen from this plot that the first PC (which accounts for $84.75 \%$ of the total explained variance (TEV)) captures the variation caused by different concentrations of spiked Sudan-1 and the second PC (which only accounts for $4.16 \%$ TEV) captures the minor experimental variations. The PCA loadings plot from the first principal component (Figure 4B) clearly shows that many spectral features relating to Sudan-1 (Figure 1) are important for this quantitative separation in PC1. It is noteworthy that the clusters of high concentrations show some overlap, however the separation improves as the concentration of the Sudan-1 decrease; in addition, the separation with respect to Sudan-1 concentration is not wholly explained in the first PC and the second one also has some minor influence as a parabolic trajectory from low to high concentration in both $\mathrm{PC} 1$ and $\mathrm{PC} 2$
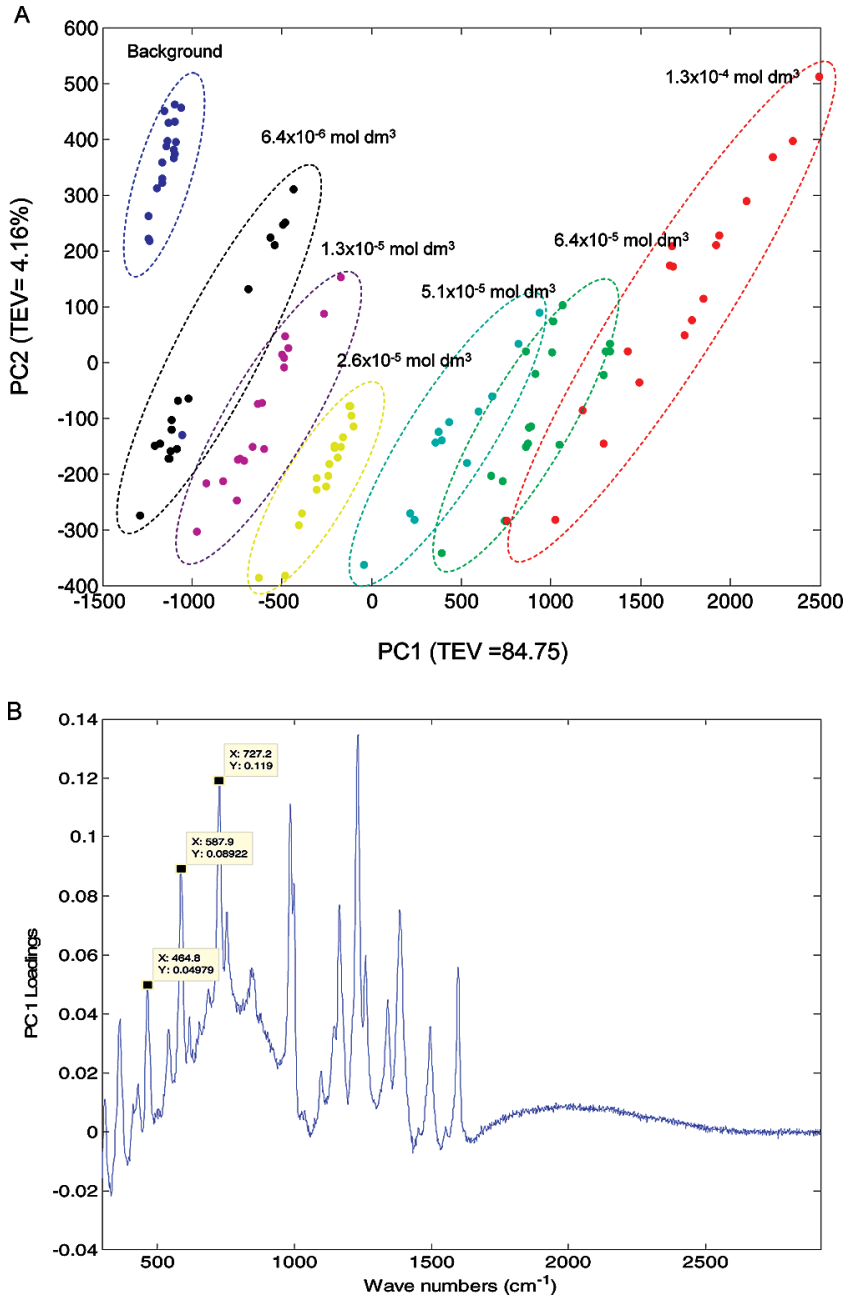

Figure 4. (A) PCA score plot from SERS spectra acquired from spiking Sudan-1 into chilli powder. The ellipses are drawn as a guide and have no statistical meaning. (B) The corresponding PCA loadings plot from the first principal component illustrating which spectral features are important for separation in PC1; highlighted are the three peaks used for univariate quantification.

(Figure 4). In combination this suggests that the SERS response of Sudan-1 may be nonlinear, particularly at high concentrations. Nevertheless, the results from the unsupervised PCA suggests that it is possible to use multivariate regression models to model the relationship between the SERS response of Sudan-1 and it concentration level in the chilli extract using the whole Raman spectrum.

Multivariate Regression. We therefore constructed multivariate models using PLS regression, $\varepsilon$-SVR and ANNs, and Figure 5 shows the predictions from the test data only and this clearly shows that the predictive ability of all three models (Figure 5a-c) was excellent as these predictions lie on the expected $y=x$ line.

We also made comparisons between the univariate regression and multivariate regressions by performing the same type of cross-validation on both the univariate and multivariate regression. The RMSECV and $Q^{2}$ obtained from the double crossvalidation as described in data analysis section of the univariate regressions on 3 selected vibration bands are also shown in Table 1 along with the same metrics for PLS regression, $\varepsilon$-SVR and ANNs.

From the prediction metrics we can see that two nonlinear multivariate methods (viz., $\varepsilon$-SVR and ANNs) obtained better results compared with those obtained by using the linear 
(a)

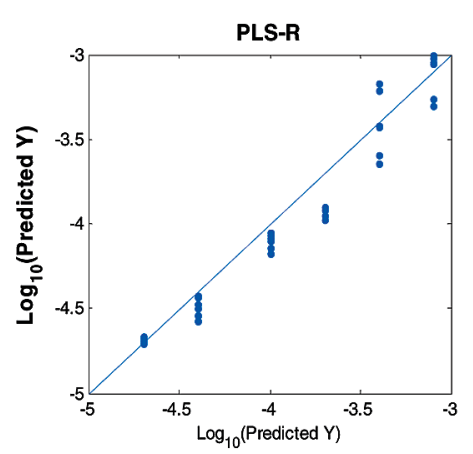

(d)

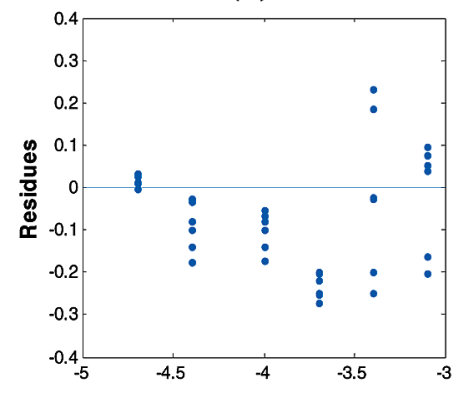

(b)

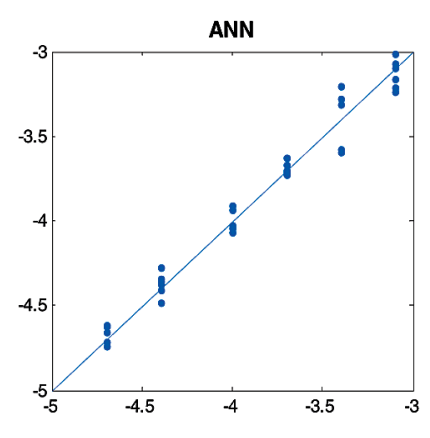

(e)

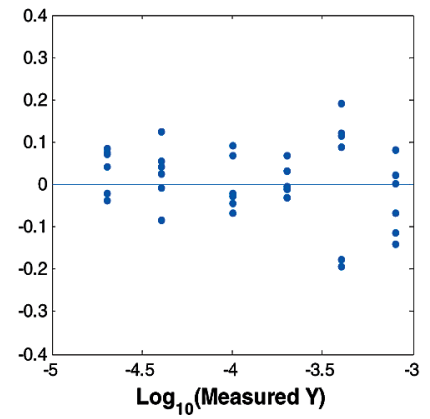

(c)

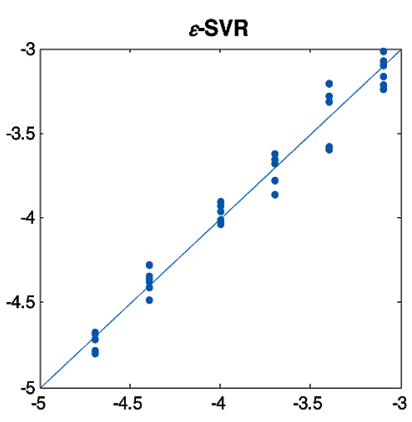

(f)

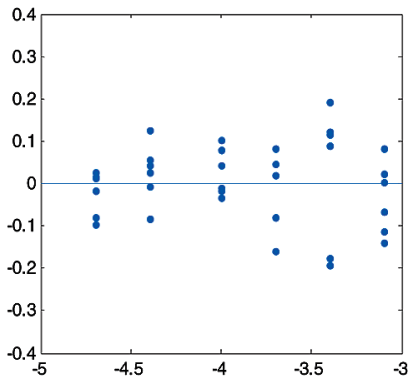

Figure 5. Plots of PLS-R results of quantification of Sudan-1 and residual plots. $(\mathrm{a}-\mathrm{c})$ Predicted concentrations against actual concentrations using PLS-R, ANN and $\varepsilon$-SVR respectively; $(\mathrm{d}-\mathrm{f})$ Plots of the residues (i.e., the difference between the actual concentrations and the predicted concentrations) against the actual concentrations from the same three models. Note the predictions do not include any dilution factors and refer to the $\log _{10}$ of concentration of Sudan-1 in the stock solution; after dilution the real concentrations are 15.56 times more dilute.

regression methods of PLS and AUC regressions. The results of ANN were the average from 10 independent multilayer perceptrons since even under exactly the same parameter setting ANN still gives (slightly) different results from different runs due to its approximation methodology. ${ }^{43,44}$ The results from PLS is marginally better than that from the univariate regression on the vibration band at $464.91 \mathrm{~cm}^{-1}$ while the results of univariate regression on the vibration bands at $587.79 \mathrm{~cm}^{-1}$ and 727.23 $\mathrm{cm}^{-1}$ are the poorest, probably due to the fact that these bands were partially overlapped with neighboring bands and hence resulted in larger integration error when calculating the AUCs. This further justifies the needs of using multivariate regression for calibration. From the residue plots (Figure $5 d-f$ ) it can be seen that the residues of the linear regression method PLS are not all distributed around 0 while for other 2 nonlinear regression methods, $\varepsilon$-SVR and ANN, the residues are mostly distributed around 0 . This further suggests that nonlinearity exists in the relation between the SERS response and the concentration level of the analyte.

\section{Conclusions}

A gold citrate reduced sol was employed in this study as it is recognized to be more homogeneous compared to silver sols. ${ }^{45}$ The results obtained show that this is viable as a suitable substrate for semiquantitative analysis of Sudan-1, as good correlations coefficients of $R^{2}=0.985,0.965$, and 0.979 were observed for the vibrational bands at 464.91, 587.79, and 727.23 $\mathrm{cm}^{-1}$ respectively and with standard deviations of only $13.3 \%$.

The recent introduction of portable Raman spectrometers for chemical analysis, in situ, and the widespread interest of Raman spectroscopy require that investigations be made to determine the sensitivity of such instrumentation. Although portability is a distinct advantage this has only been shown to allow for qualitative analysis of analytes. However the enormous interest in applying the SERS technique to both qualitative and quantitative chemical analysis requires that tangible working models be available to demonstrate the viability of compact/ portable Raman spectrometers. The data reported herein demonstrated that not only is it possible to get good sensitivity by employing the SERS technique using a portable Raman spectrometer (an Ahura defender system), but that multivariate chemometrics is essential for providing excellent sensitivity and quantitative data compared to classical univariate area under specific vibrational bands approaches. The limit of detection of Sudan-1 spiked into a complex matrix is calculated as $48 \mu \mathrm{g} /$ $\mathrm{kg}$ chilli powder. This is currently an order higher than HPLCbased approaches which use considerable sample clean up prior to analysis, ${ }^{9,10}$ and an area of work will be to optimize analyte extraction from the sample matrix prior to SERS.

Acknowledgment. I.T.S. and R.G. are very thankful to UK BBSRC for funding and Y.X. and R.G. are grateful to the European Commission's BIOTRACER Integrated Project (www. biotracer.org) for financial support.

\section{References and Notes}

(1) Moller, P.; Wallin, H. Mutat. Res. 2000, 462, 13.

(2) Martinek, V.; Stiborov, M. Collect. Czech. Chem. Commun. 2002, $67,1883$.

(3) Stiborova, M.; Martinek, V.; Rydlova, H.; Hodek, P.; Frei, E. Cancer Res. 2002, 62, 5678.

(4) Garner, R. C.; Martin, C. N.; Clayson, D. B. Carcinogenic aromatic amines and related compounds. Searle, C. E., Ed.; Chemical Carcinogens, 3rd ed.; ACN Monograph 182; American Chemical Society: Washington, DC, 1984; Vol. 1.

(5) Westmoreland, C.; Gatehouse, D. G. Carcinogenesis (Lond.) 1991, 12, 1403 .

(6) Childs, J. J.; Clayson, D. S. Biochem. Pharmacol. 1966, 15, 1247. 
(7) Marmion, D. M. Handbook of US colorants for foods; John Wiley: New York, 1979

(8) Meiju, D. U.; Xiaogang, H.; Zihao, Z.; Shouguo, W. Food Chem. 2007, 105, 883-888. 465.

(9) Daood, H. G.; Biacs, M. A. J. Chromatogr. Sci. 2005, 43, 461-

(10) Sproll, C.; Ruge, W.; Strichow, N.; Attig, D.; Marx., G. Deutsche Lebensmittel-Rundschau 2005, 101, 481-484.

(11) Pielesz, A.; Baranowska, I.; Rybak, A.; Wlochowicz, A. Ecotoxicol. Environ. Safety 2002, 53, 42.

(12) Gao, X.; Liu, H.; Song, Z.; He, X.; Dong, F. Spectrosc.-Int. J 2007, 21,135

(13) DiDonna, L.; Maiuolo, L.; Mazzotti, F.; Luca, D. D.; Sindona, G. Anal. Chem. 2004, 76, 5104-5108.

(14) Tateo, F.; Bononi, M. J. Agric. Food Chem. 2004, 52, 655

(15) Zhang, Y. P.; Zhang, Y. J.; J.; Gong, W.; Gopalan, A. I.; Lee, K. P. J. Chromatogr. A 2005, 1098, 183.

(16) Calbiani, F.; Elviri, M. C. L.; Mangia, A.; Pistara, L.; Zagnoni, I. J. Chromatogr. A 2004, 1042, 123.

(17) Puoci, F.; Iemma, C. G. F.; Muzzalupo, R.; Spizzirri, U. G.; Picci, N. Food Chem. 2005, 93, 349.

(18) Chalmers, J. M.; Griffiths., P. R. Handbook of Vibrational Spectroscopy; John Wiley \& Sons, Ltd.: Chichester, U.K., 2002; Vol. 1.

(19) Ferraro, J. R.; Nakamoto. K. Introductory Raman Spectroscopy; Academic Press: London, 1994.

(20) Fleischmann, M.; Hendra, P. J.; McQuillan, A. J. Chem. Phys. Lett. 1974, 26, 163 .

(21) Moskovits, M. Rev. Mod. Phys. 1985, 57, 783.

(22) Jarvis, R. M.; Goodacre, R. Chem. Soc. Rev. 2008, 37, 931.

(23) Graham, D.; Faulds, K. Chem. Soc. Rev. 2008, 37, 1042.

(24) Kneipp, K.; Kneipp, H.; Tzkan, I.; Dasari, R. R.; Feld, M. S.; Dresselhaus, M. S. Nonlinear Raman probe of single molecules attached to colloidal silver and gold clusters. Optical Properties of Nanostructured Random Media; Springer-Verlag: Berlin, 2002; Vol. 82; p 227.

(25) Moskovits, M.; Tay, L. L.; Yang, J.; Haslett, T. SERS and the single molecule. Opt. Prop. Nanostruct. Random Media 2002, 82, 215.

(26) Brereton, R. G. Cheometrics Data analysis for the laboratory and chemical plants.; Wiley: Chichester, U.K., 2002.

(27) Massart, D. L.; Vandeginste, B. G. M.; Deming, S. N.; Michotte, Y.; Kaufman., L. Chemometrics: A Textbook; Elsevier Science Publishers B V: Netherlands, 1988.
(28) Boelens, H. F. M.; Djikstra, R. J.; Eilers, P. H. C.; Fitzpatrick, F.; Westerhuis, J. A. J. Chromatogr. A 2004, 1057, 21.

(29) Wold, H. In Multivariate Analysis; Krishnaiah, K. R., Ed.; Academic Press: New York, 1966; p 391.

(30) Beavis, R. C.; Colby, S. M.; Goodacre; R. Harrington; P. Reilly; J. P. Solokolow, S.; Wilerson., C. W. Encyclopedia of Analytical Chemistry; Meyers, R. A., Ed.; Wiley: Chichester, U.K., 2000; p 11558.

(31) Alsberg, B. K.; Kell, D. B.; Goodacre, R. Anal. Chem. 1998, 70, 4126.

(32) Dixon, S. J.; Xu, Y.; Brereton, R. G.; Soini, H. A.; Novotny, M. V.; Oberzaucher, E.; Grammer, K.; Penn, D. J. Chemom. Intell. Lab. Syst. 2007, 87, 161.

(33) Cortes, C.; Vapnik, V. Machine Learn. 1995, 20, 273.

(34) McGovern, A. C.; Broadhurst, D.; Taylor, J.; Kaderbhai, N.; Winson, M. K.; Small, D. A.; Rowland, J. J.; Kell, D. B.; Goodacre., R. Biotechnol. Bioeng. 2002, 78, 527.

(35) Wasserman, P. D. Neural Computing: Theory and Practice; Van Nostrand Reinhold: New York, 1989.

(36) Cheung, W.; Xu, Y.; Thomas, C. L. P.; Goodacre, R. Analyst 2009 , 134, 557.

(37) Filzmoser, P.; Liebmann, B.; Varmuza, K. J. Chemom. 2009, 23, 160.

(38) Westerhuis, J. A.; Hoefsloot, H. C. J.; Smit, S.; Vis, D. J.; Smild, A. K.; van Velzen, E. J. J.; van Duijnhoven, J. P. M.; van Dorsten, F. A. Metabolomics 2008, 4, 81.

(39) Degen, I. A. Tables of chracteristic group frequencies for the interpretation of infrared and Raman spectra; Acolyte Publications: Harrow, U.K., 1997.

(40) Socrates, G. Infrared and Raman characteristic group frequencies: tables and charts; John Wiley and Sons: Chichester, U.K., 2001.

(41) Shadi, I. T.; Chowdhry, B. Z.; Snowden, M. J.; Withnall, R. J. Raman Spectrosc. 2004, 35, 800 .

(42) Shadi, I. T.; Cheung, W.; Goodacre, R. Anal. Bioanal. Chem. 2009, $394,1833$.

(43) Goodacre, R.; Kell, D. B.; Bianchi, G. Nature 1992, 359, 594.

(44) Goodacre, R.; Timmins, É.M.; Burton, R.; Kaderbhai, N.; Woodward, A. M.; Kell, D. B.; Rooney, P. J. Microbiology 1998, 144, 1157.

(45) Jarvis, R. M.; Johnson, H. E.; Olembe, E.; Panneerselvam, A.; Malik, M. A.; Azad, M.; O'Brien, P.; Goodacre, R. Analyst 2008, 133, 1449.

JP908892N 